https://doi.org/10.15407/ujpe64.8.710

P. MINAIEV, V. SKALOZUB

Oles Honchar Dnipro National University

(72, Gagarin Ave., Dnipro 49010,Ukraine; e-mail: minaevp9595@gmail.com)

\title{
THE ELECTROWEAK PHASE TRANSITION IN A SPONTANEOUSLY MAGNETIZED PLASMA
}

\begin{abstract}
We investigate the electroweak phase transition (EWPT) in the Minimal (One Higgs doublet) Standard Model (SM) with account for the spontaneous generation of magnetic and chromomagnetic fields. As it is known, in the SM for the mass of a Higgs boson greater than $75 \mathrm{GeV}$, this phase transition is of the second order. But, according to Sakharov's conditions for the formation of the baryon asymmetry in the early Universe, it has to be strongly of the first order. In the Two Higgs doublets SM, there is a parametric space, where the first-order phase transition is realized for the realistic Higgs boson mass $m_{\mathrm{H}}=125 \mathrm{GeV}$. On the other hand, in the hot Universe, the spontaneous magnetization of a plasma had happened. The spontaneously generated (chromo) magnetic fields are temperature-dependent. They influence the EWPT. The color chromomagnetic fields $B_{3}$ and $B_{8}$ are created spontaneously in the gluon sector of $Q C D$ at a temperature $T>T_{d}$ higher the deconfinement temperature $T_{d}$. The usual magnetic field $H$ has also to be spontaneously generated. For $T$ close to the $T_{E W P T}$, these magnetic fields could change the kind of the phase transition.

Ke ywords: electroweak phase transition, standard model, deconfinement.
\end{abstract}

\section{Introduction}

In the Early Universe, there are many phase transitions. The most important is EWPT, when particles acquired masses. Other important problem is baryogenesis.

As is well known, in the Minimal Standard Model (MSM) of elementary particles, EWPT is of the first order for the mass of a Higgs boson less than 75 $\mathrm{GeV}$. For greater masses, it is of the second order. Experiments give $m_{\mathrm{H}}=125 \mathrm{GeV}$. Sakharov [1] proposed the conditions for generation of the asymmetry between baryons and antibaryons. Today, they are formulated as three baryogenesis conditions. According to them, the phase transition should be strongly of the first order. So, Sakharov's conditions are violated.

Another important property of non-Abelian gauge fields at high temperatures is a spontaneous vacuum magnetization. It is closely related to the asymptotic freedom, which happens due to a large magnetic moment of charged color gluons (gyromagnetic ratio $\gamma=2)$.

In fact, the asymptotic freedom at high temperatures is always accompanied by the background sta-

(C) P. MINAIEV, V. SKALOZUB, 2019 ble, temperature-dependent, and long-range chromomagnetic fields [2].

The magnetization phenomenon was investigated in the $\mathrm{SU}(3)$ gluodynamics in detail [3], and the supersymmetric theories $[4,5]$ were developed by analytic methods and in the $\mathrm{SU}(2)$ gluodynamics $[6,7]$ by Monte-Carlo simulations on a lattice. In all these cases, the spontaneous creation of magnetic fields was bdetected. Within the application to the early Universe, the spontaneous vacuum magnetization in the electroweak sector of the standard model was described in [8].

At the LHC experiments, a new matter, namely, phase-quark-gluon plasma (QGP), has to be produced in heavy ion collisions. The deconfinement phase transition (DPT) temperature is expected to be of the order of $T_{d} \sim 180-200 \mathrm{MeV}$. In theory, the investigation of the DPT and QGP properties were carried out by different method - analytic perturbative and nonperturbative.

In papers $[9,10]$, we have shown that, due to the vacuum polarization of quark fields by the color magnetic fields $B_{3}$ and $B_{8}$ existing in the QGP after DPT, the usual magnetic field $H$ can be generated for temperatures $T_{d}<T<T_{\text {EWPT. The field } H \text { is }}$

ISSN 2071-0194. Ukr. J. Phys. 2019. Vol. 64, No. 8 
temperature-dependent and occupies a large plasma volume as the fields $B_{3}$ and $B_{8}$ [3].

In the present paper, we will investigate the influence of the magnetic and chromomagnetic fields spontaneously created after DPT on EWPT. The magnetic fields could realize Sakharov's conditions in MSM and change the behavior of phase transitions as in the superconductivity. The proper time representation is used. The effective potential of the external fields $V\left(\phi, B_{3}, B_{8}, H, T\right)$ with one-loop plus daisy diagrams accounting for the gluons and all quark flavors at finite temperatures is calculated. This field configuration is stable due to the daisy diagram contributions, which cancel the imaginary terms presenting in the one-loop effective potential of charged gluons $V^{(1)}\left(B_{3}, B_{8}, T\right)$. To estimate the field strengths, the asymptotic high temperature expansion derived by Mellin's transformation technique is applied $[2,11]$.

\section{Effective Potential of MSM with Magnetic Fields at Finite Temperatures}

The spontaneous vacuum magnetization has been derived from the investigation of the effective potential (EP) of covariantly constant(soursless) chromomagnetic fields $H^{a}=H \delta^{a 3}$, which is a solution to the classical Yang-Mills field equation, where $H=$ const, and $a$ is an isotopic index,

$V(H, T)=\frac{H^{2}}{2}+V^{(1)}(H, T)$.

It includes the tree-level and one-loop parts. The minimum of the EP at a high temperature $T$ corresponds to the nonzero magnetic field.

The Lagrangian of the boson sector of the SalamWeinberg model is

$$
\begin{aligned}
& L=-\frac{1}{4} F_{\mu \nu}^{a} F_{a}^{\mu \nu}-\frac{1}{4} G_{\mu \nu} G^{\mu \nu}+\left(D_{\mu} \Phi\right)^{+}\left(D^{\mu} \Phi\right)+ \\
& +\frac{m^{2}}{2}\left(\Phi^{2}+\Phi\right)-\frac{\lambda}{4}\left(\Phi^{2}+\Phi\right)^{2},
\end{aligned}
$$

where the following standard notations are introduced:

$$
\begin{aligned}
& F_{\mu \nu}^{a}=\partial_{\mu} A_{\nu}^{a}-\partial_{\nu} A_{\mu}^{a}+g \varepsilon^{a b c} A_{\mu}^{b} A_{\nu}^{c}, \\
& G_{\mu \nu}^{a}=\partial_{\mu} B_{\nu}-\partial_{\nu} B_{\mu}, \\
& D_{\mu}=\partial_{\mu}+\frac{1}{2} i g A_{\mu}^{a} \tau^{a}+\frac{1}{2} i g^{\prime} B_{\mu} .
\end{aligned}
$$

ISSN 2071-0194. Ukr. J. Phys. 2019. Vol. 64, No. 8
The vacuum expectation value of the field $\Phi$ is

$$
\langle\Phi\rangle=\frac{1}{\sqrt{2}}\left(\begin{array}{c}
0 \\
\phi_{c}
\end{array}\right) \text {. }
$$

The fields of $Z$-, $W^{ \pm}$-bosons, and photons are

$W_{\mu}^{ \pm}=\frac{1}{\sqrt{2}}\left(A_{\mu}^{1} \pm i A_{\mu}^{2}\right)$

$Z_{\mu}=\frac{1}{\sqrt{g^{2}+g^{\prime 2}}}\left(g A_{\mu}^{3}-g^{\prime} B_{\mu}\right)$

$A_{\mu}=\frac{1}{\sqrt{g^{2}+g^{\prime 2}}}\left(g^{\prime} A_{\mu}^{3}+g^{\prime} B_{\mu}\right)$.

For the investigation of EWPT, according to [9,13], the EP is

$V\left(H, T, \phi_{c}\right)=\frac{H_{8}^{2}}{2}+\frac{H_{3}^{2}}{2}+\frac{H^{2}}{2}+$

$+V^{(0)}\left(\phi_{c}\right)+V^{(1)}\left(H, T, \phi_{c}\right)+V^{(\text {Ring })}\left(H, T, \phi_{c}\right)$.

To compute the EP $V^{(1)}$ in the background magnetic fields, let us introduce the proper time, and $s$ representation for the Green functions:

$G^{a b}=-i \int \exp \left(-i s\left(G^{-1}\right)^{a b}\right) d s$.

To incorporate the temperature into this formalism, the connection between the Matsubara Green function and the Green function at the zero temperature is needed:

$G_{k}^{a b}\left(x, x^{\prime} ; T\right)=$

$=\sum_{-\infty}^{+\infty}(-1)^{(n+[x]) \sigma_{k}} G_{k}^{a b}\left(x-[x] \beta u, x^{\prime}-n \beta u\right)$,

where $G_{k}^{a b}$ is the corresponding function at $T=0$, $\beta=1 / T, u=(0,0,0,1),[x]$ denotes an integer part of $x_{4} / \beta, \sigma_{k}=1$ in the case of physical fermions, and $\sigma_{k}=0$ for bosons and ghost fields.

The one-loop contribution to EP is given by the expression

$V^{(1)}=-\frac{1}{2} \operatorname{Tr} \ln G^{a b}$,

where $G^{a b}$ stands for the propagators of all the quantum fields $W^{ \pm}, Z, \ldots$ in the background magnetic field $H$.

The term with $n=0$ in Eqs. (8) and (9) gives the zero-temperature expression for the Green function and EP, respectively. 

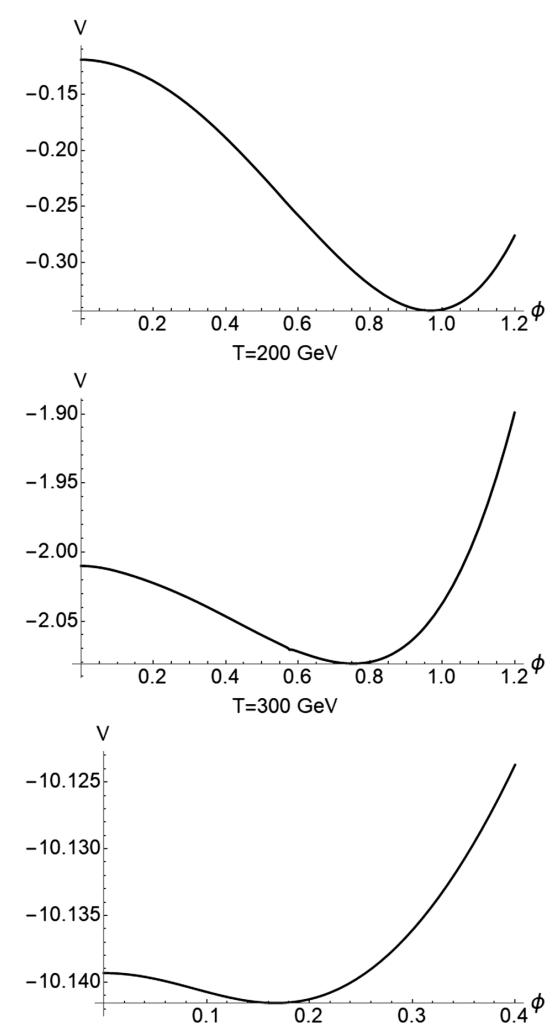

Fig. 1. Effective potential view at different temperatures; the symmetry is broken

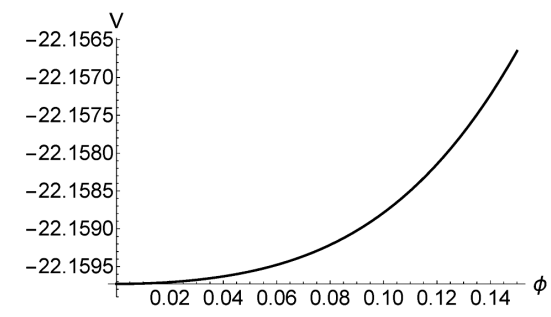

Fig. 2. Effective potential view at a temperature of $365 \mathrm{GeV}$; the symmetry is restored

Strengths of generated fields

\begin{tabular}{|c|l|c|c|c|c|}
\hline$T, \mathrm{GeV}$ & $\phi$ & $V$ & $H, 10^{21} \mathrm{G}$ & $H_{3}, 10^{23} \mathrm{G}$ & $H_{8}, 10^{23} \mathrm{G}$ \\
\hline 100 & 0.96 & -0.35 & 0.436 & 0.131 & 1.092 \\
200 & 0.75 & -2.1 & 1.97 & 0.601 & 3.17 \\
260 & 0.4 & -4.9 & 3.28 & 0.928 & 4.91 \\
300 & 0.26 & -10.14 & 4.70 & 1.20 & 6.66 \\
350 & 0.11 & -18.77 & 6.66 & 2.13 & 9.28 \\
363 & 0.01 & -21.68 & 6.69 & 2.18 & 9.51 \\
\hline
\end{tabular}

In the quark sector of EP, we have the mixing of external magnetic and chromomagnetic fields, according to [9]. The next linear combinations are appear

$$
\left\{\begin{array}{l}
\mathcal{H}_{f}^{1}=q_{f} H+g\left(\frac{H_{3}}{2}+\frac{H_{8}}{2 \sqrt{3}}\right) \\
\mathcal{H}_{f}^{2}=q_{f} H+g\left(\frac{H_{8}}{2 \sqrt{3}}-\frac{H_{3}}{2}\right) \\
\mathcal{H}_{f}^{3}=q_{f} H-g \frac{H_{8}}{\sqrt{3}} .
\end{array}\right.
$$

They are included in the quark part of EP

$$
\begin{aligned}
& V_{\text {quark }}=\frac{1}{8 \pi^{2}} \sum_{f} \sum_{a=1}^{3} \sum_{l=-\infty}^{\infty}(-1)^{l} \times \\
& \times \int_{0}^{\infty} \frac{d s}{s^{3}} e^{-m_{f}^{2} s-\frac{\beta^{2} l^{2}}{4 s}}\left(s \mathcal{H}_{f}^{a} \operatorname{coth}\left(s \mathcal{H}_{f}^{a}\right)-1\right) .
\end{aligned}
$$

In our calculations, $H, H_{3}$, and $H_{8}$ are parameters. To investigate EWPT, we need to calculate EP as a function of $\phi_{c}$ at some constant temperature and for different temperatures, to look after the behavior of the symmetry, and to find the values of parameters, which minimize EP.

\section{Numerical Results}

For numerical calculations, we use the following dimensionless parameters:

$$
\begin{gathered}
V^{0}=\frac{V^{(0)} e^{2}}{M_{W}^{4}} ; \quad V^{T}=\frac{V^{(T)} e^{2}}{M_{W}^{4}} ; \quad \phi=\frac{\phi_{c}}{\delta(0)} ; \\
\mu_{f}=\frac{m_{f}}{M_{W}} ; \quad h_{f, a}=\frac{e \mathcal{H}_{f}^{a}}{M_{W}^{2}} ; \quad \beta_{p}=M_{W} \beta .
\end{gathered}
$$

After the calculation, we should find the minimum value depending on $\phi, H_{3}, H_{8}$, and $H$ for a fixed temperature.

The strength of generated fields at the energy minimum is shown in Table. The most important point is the next one - we have nonzero chromomagnetic and magnetic fields and a negative value of EP. The magnetic field is two orders less than the chromomagnetic one.

In Figs. 1 and 2, the behavior of the symmetry is shown. We have minimum of EP with a nonzero scalar field. The symmetry is restored at high temperatures. The critical temperature is obtained near $T_{\mathrm{EWPT}}=365 \mathrm{GeV}$.

ISSN 2071-0194. Ukr. J. Phys. 2019. Vol. 64, No. 8 


\section{Conclusions}

In our calculations, we applied the consistent approximation for the effective potential accounting for the one-loop plus daisy diagrams. It includes the terms of the order $\sim g^{2}$ and $\sim g^{3}$ and makes the potential real due to the cancellation of the imaginary terms. That is sufficient at high temperatures because of small couplings.

The most interesting observation of the above investigation is twofold. First, as the temperature grows, the magnetic field strengths are increased. Second, simultaneously, the value of the effective potential at the minimum is negative.

Really, as we have noted already, the asymptotic freedom at high temperatures has always to be accompanied by the temperature-dependent background magnetic fields [2].

As it follows from the obtained results, the strong chromo(magnetic) fields of the order $H_{3.8} \sim 10^{18}$ $10^{19} \mathrm{G}$ and $H \sim 10^{16}-10^{17} \mathrm{G}$ must be present in QGP [9]. This influences all the processes happening and may serve as the distinguishable signals of DPT. Due to the magnetization, in particular, all the initial states of charged particles have to be discrete ones. These fields are present at higher temperatures, as the deconfinement appears. At temperatures close to $T_{\text {EWPH }}$, the strengths are 5 order higher than for the deconfinement temperature.

We have demonstrated that EWPT in MSM has the critical temperature near $360 \mathrm{GeV}$, and the nonzero magnetic and chromomagnetic fields should be spontaneously generated as well. In Fig. 1, we see that there is no reheating phase. This illustrates that the phase transition is of the second type, and Sakharov's conditions are not satisfied. So, even with magnetic and chromomagnetic fields, the phase transition is of the second order.

As was shown in [12], the Sakharov conditions can be satisfied in the parametric space of the Two Higgs doublet Standard Model without background magnetic fields.

This work is partially supported by the ICTP through AF-06.

1. A.D. Sakharov. Violation of CP Invariance, C asymmetry, and baryon asymmetry of the universe. JETP Lett. 5, 32 (1967).

2. V. Skalozub. Effective coupling constants in gauge theories at high temperature. Int. J. Mod. Phys. A 115643 (1996).
3. V. Skalozub, A. Strelchenko. On the generation of Abelian magnetic fields in $\mathrm{SU}(3)$ gluodynamics at high temperature. Eur. Phys. J. C 33, 105 (2004).

4. M.D. Pollock. Magnetic fields and vacuum polarization at the Planck era. Int. J. Mod. Phys. D 12, 1289 (2003).

5. V.I. Demchik, V.V. Skalozub. The spontaneous generation of magnetic fields at high temperature in a supersymmetric theory. Eur. Phys. J. C 27, 601 (2003).

6. V. Demchik, V. Skalozub. The spontaneous creation of a chromomagnetic field and $A_{0}$-condensate at high temperature on a lattice. J. Phys. A 41, 164051 (2008).

7. S. Antropov, M. Bordag, V. Demchik, V. Skalozub. Long range chromomagnetic fields at high temperature. Intern. J. Mod. Phys. A 26, 4831 (2011).

8. V. Demchik, V. Skalozub. Spontaneous magnetization of a vacuum in the hot Universe and intergalactic magnetic fields. Phys. Part. Nucl. 46, 1 (2015).

9. V. Skalozub, P. Minaiev. Magnetized quark-gluon plasma at the LHC. Phys. Part. Nucl. Lett. 15, 568 (2018).

10. V. Skalozub, P. Minaiev. On magnetization of quarkgluon plasma at the LHC experiment energies. Visnyk Dnipropetr. Univ. Fiz. Radioel. 24, (2016).

11. H.E. Haber, H.A. Weldon. On the relativistic BoseEinstein integrals. J. Math. Phys. 23, 1852 (1982).

12. V. Skalozub, A. Kozhushko. The parametric space of the two-Higgs-doublet model and Sakharov's baryogenesis conditions. Ukr. J. Phys. 56, 431 (2011).

13. V. Skalozub, V. Demchik. Electroweak phase transition in strong magnetic fields in the standard model of elementary particles. arXiv:hep-th/9912071v1 (1999).

Received 08.07.19

\section{П. Мінаєв, В. Скалозуб}

\section{ЕЛЕКТРОСЛАБКИЙ ФАЗОВИЙ}

\section{ПЕРЕХІД В СПОНТАННО НАМАГНІЧЕНІЙ ПЛАЗМІ}

$\mathrm{P}$ е $з$ ю м е

Досліджується електрослабкий фазовий перехід в мінімальній (один дуплет хіггсівських бозонів) Стандартній Моделі (CM) з урахуванням спонтанного народження магнітних та хромомагнітних полів при високій температурі. Як відомо, в СМ для маси бозона Хіггса, більшій за 75 ГеВ, цей фазовий перехід є переходом другого роду. Але відповідно до критеріїв Сахарова для формування баріонної асиметрії на ранніх етапах еволюції Всесвіту, він повинен бути жорстким переходом першого роду. В параметричному просторі дводуплетної СМ без магнітних полів можливий перехід першого роду. В ранньому Всесвіті існували спонтанно народжені температурозалежні магнітні та хромомагнітні поля. Хромомагнітні поля $B_{3}$ і $B_{8}$ народжувались в глюонному секторі КХД за температури $T>T_{d}$, більшої за температуру деконфайменту $T_{d}$. Звичайне магнітне поле народжувалось за рахунок кваркових петель. Як результат, для температур $T$, близьких до критичної температури $T_{\mathrm{EwPT}}$, ці поля можуть змінити характер фазового переходу. 